\title{
Accelerating the Additive Revolution
}

\author{
EDWARD D. HERDERICK (1) ${ }^{1}$ \\ 1.-GE Inspection Technologies, Cincinnati, OH, USA
}

The past year has seen accelerated growth and adoption of additive manufacturing (AM) by several measures, most notably in industrial adoption and implementation for materials intensive applications. Per the Wohlers Report 2016, the AM industry, consisting of all AM products and services worldwide, grew $25.9 \%$ (CAGR) to a total of US $\$ 5.165$ billion in $2015 .^{1}$ Further, sales of AM systems for manufacturing metal parts grew at a year-on-year rate of $46.9 \%$ for the most recent data available. ${ }^{1}$ This is reflective of greater interest and acceptance of the industrial manufacturing community for AM as a standard technique.

This acceptance is the product of over 25 years of intensive research and development. Two early works of note include the first paper describing powder bed fusion AM in JOM in $1990,{ }^{2}$ in this case a polymeric wax and ABS materials. Similarly, the first description of using powder bed fusion AM for metals in Metallurgical and Materials Transactions $A$ appeared in $1993,{ }^{3}$ in this case using intermetallic nickel-tin powder. Comparison of these early papers to recently published work ${ }^{4}$ documenting fatigue evaluation of Ti-6Al-4V and nickel alloy 625 , produced using commercially available powders and commercially available equipment, shows the progress that the materials processing community has driven and which serves as the foundation for accelerating growth in applications.

The bedrock for this growth is the deeper understanding of the entire value stream for producing AM parts. For metal part production in the traditional manufacturing paradigm, a foundry provides material specifications to original equipment manufacturers consisting of the raw feedstock specification, cast properties, and forged ingot properties as applicable. In the new AM paradigm, the process looks quite different on first review. However, on deeper evaluation, the metal additive process qualification route follows the standard material intensive

Edward D. Herderick is the JOM advisor for the Process Technology \& Modeling Committee of the TMS Extraction \& Processing Division, and guest editor for the topic Progress in Additive Manufacturing in this issue. manufacturing route with specific elements modified per the process itself. The supplied powder is certified to specification, a fixed process is qualified, mechanical and chemical properties of interest are measured for the specific application, and an ongoing monitor for compliance is established. Post-machining, thermal processing, and non-destructive inspection and testing protocols specific to performance requirements are implemented just as in a performanceintensive weldment or cast component application. A critical role for the minerals, metals, and materials community is exploring and evaluating elements of the total process value stream and impacts on the microstructure and defect populations in relation to performance. Further, new characterization and non-destructive inspection methods tailored for complex AM-produced structures will be a key contribution by the community.

The additive revolution is clearly accelerating, as evidenced by the growth in investment and industrial applications. As of this writing at the close of 2016, 40 CFM International LEAP-1A engines with AM-produced fuel nozzles are in active revenue service with many hundreds more to enter service in $2017 .{ }^{5}$ Other new applications continue to emerge that are transforming engine architecture using AM. One example is the core main injector for the Aerojet Rocketdyne RL10 upper-stage rocket engine, which was printed using metal powder bed fusion and successfully tested in conjunction with the USAF and NASA. ${ }^{6}$ Another example is the GE Aviation Advanced Turbo Prop engine that has no structural castings and consolidated over $855 \mathrm{sub}-$ tractively-built parts into 12 printed parts by applying designs for AM. ${ }^{7}$ Example applications beyond aerospace include downhole tooling in the oil and gas industry ${ }^{8}$ and spinal implants in the medical device industry. ${ }^{9}$ GE Additive has recently announced the goal of deploying 10,000 metal AM machines over the next 10 years for customers around the world to accelerate the additive revolution. ${ }^{10}$ These industrial investments show commitment to growing the applications for AM across many industrial sectors. 
In the spirit of providing the technical foundation for accelerating growth of AM, it is a great pleasure to present the 2017 follow-up installment to the special topic Progress in Additive Manufacturing. ${ }^{11,12}$ Taken together, the papers here demonstrate deeper understanding of the depth and breadth of the AM field. They present work on a cross-section of processing routes including laser powder bed fusion, electron beam powder bed fusion, powder bed binder jetting, ultrasonic additive manufacturing, and fused deposition modeling.

Seifi and authors present a review of standards activities in support of the qualification and certification of AM-built components. Holm and authors demonstrate an approach for utilizing computer vision and machine learning to evaluate AM feedstocks. Qian and authors measure the mechanical properties of AM-built material and the effects of post-processing. Horn and authors explore the effects of alloying additions on AM-built material. Cunningham and authors characterize AM-built material using advanced computed tomography. Hehr and authors demonstrate an industrial application for ultrasonic additive manufacturing. Manogharan and authors show an optimization route for powder bed binder jetting. Finally, Ahmed and authors investigate the mechanical properties of AM-built polymers.

The future for additive manufacturing is bright. Today, it is an exciting technology with a relatively limited number of materials and process combinations. It will be up to the community to apply fundamental physical metallurgical principles and materials-intensive processing fundamentals to unlock its full potential and continue to accelerate the additive revolution.

To download any of the papers being published under the topic of Progress in Additive Manufacturing, follow the url http://link.springer.com/jour nal/11837/69/3/page/1 to the table of contents page for the March 2017 issue (vol. 69, no. 3).

- "Metal Additive Manufacturing Standardization in Support of Qualification and Certification" by Mohsen Seifi, Michael Gorelik, Jess Waller, Nik Hrabe, Nima Shamsaei, Steve Daniewicz, and John J. Lewandowski.

- "Computer Vision and Machine Learning for Autonomous Characterization of AM Powder Feedstocks" by Brian L. DeCost, Harshvardhan Jain, Anthony D. Rollett, and Elizabeth A. Holm.

- "Additive Manufacturing of Ti-6Al-4V Sheet" by H.P. Tang, J. Wang, C.N. Song, N. Liu, L. Jia, and M. Qian.

- "Effect of Hypoeutectic Boron Additions on the Grain Size and Mechanical Properties of Ti-6Al-
4V Manufactured Using Powder Bed Electron Beam Additive Manufacturing" by Zaynab Mahbooba, Harvey West, Ola Harrysson, Andrzej Wojcieszynski Ryan Dehoff, Peeyush Nandwana, and Timothy Horn.

- "Synchrotron-Based x-ray Microtomography Characterization of the Effect of Processing Variables on Porosity Formation in Laser Power-Bed Additive Manufacturing of Ti-6Al$4 \mathrm{~V}$ " by Ross Cunningham, Sneha P. Narra, Colt Montgomery, Jack Beuth, and A.D. Rollett.

- "Five-Axis Ultrasonic Additive Manufacturing for Nuclear Component Manufacture" by Adam Hehr, Justin Wenning, Kurt Terrani, Sundarsanam Suresh Babu, and Mark Norfolk.

- "Optimization of Binder Jetting Using Taguchi Method" by Sanjay Shrestha and Guha Manogharan.

- "Investigation on the Flexural Creep Stiffness Behavior of PC-ABS Material Processed by Fused Deposition Modeling Using Response Surface Definitive Screening Design" by Omar Ahmed Mohamed, Syed Hasan Masood, and Jahar Lal Bhowmik.

\section{REFERENCES}

1. Wohlers Report 2016, 3D Printing and Additive Manufacturing State of the Industry, Annual Worldwide Progress Report, ISBN 978-0-9913332-2-6. Executive Summary, p. 3.

2. H.L. Marcus, J.J. Beaman, J.W. Barlow, and D.L. Bourell, JOM 42, 8 (1990).

3. W.L. Weiss and D.L. Bourell, Metall. Mater. Trans. A 24A, 757 (1993).

4. D.B. Witkin, T.V. Albright, and D.N. Patel, Metall. Mater. Trans. A 47A, 3823 (2016).

5. "Over 40 Leap-1A engines delivered: Safran," https://www. flightglobal.com/news/articles/over-40-leap-1a-engines-de livered-safran-430698/. Accessed 5 January 2017.

6. "Aerojet Rocketdyne Successfully Tests Complex 3-D Printed Injector in World's Most Reliable Upper Stage Rocket Engine," http://www.rocket.com/article/aerojet-rock etdyne-successfully-tests-complex-3-d-printed-injectorworlds-most-reliable. Accessed 6 January 2017.

7. "GE tests additive manufactured demonstrator engine for Advanced Turboprop," http://www.geaviation.com/press/ business_general/bus_20161031a.html. Accessed 5 January 2017.

8. "Metal Additive Manufacturing used for production of large scale downhole tool for Oil \& Gas sector," http://www. metal-am.com/metal-additive-manufacturing-for-oil-and-gassector/. Accessed 6 January 2017.

9. "Additive Manufacturing for Spinal Implants from Concept Laser," http://www.tctmagazine.com/3D-printing-news/ad ditive-manufacturing-for-spinal-implants-from-conceptlase/. Accessed 5 January 2017.

10. "How GE Additive Will Accelerate the 3D Printing Revolution," http://www.tctmagazine.com/how-ge-additive-plansaccelerate-3d-printing-revolution/. Accessed 5 January 2017.

11. E.D. Herderick, JOM 67, 580 (2015).

12. E.D. Herderick, JOM 68, 721 (2016). 